\title{
The Role of Exercise in the Quality of Life in Patients After Pancreatectomy: A Prospective Randomized Controlled Trial
}

\author{
Anastasios Katsourakis $^{\mathrm{a}, \mathrm{e}}$, Ioannis Vrabas ${ }^{\mathrm{b}}$, Vassilios Papanikolaou, \\ Stylianos Apostolidis ${ }^{\mathrm{d}}$, Iosif Chatzis ${ }^{\mathrm{a}}$, George Noussios ${ }^{\mathrm{b}}$
}

\begin{abstract}
Background: Pancreatic resection is still a challenging operation characterised by high morbidity. The quality of life in patients after pancreatectomy is a critical outcome. The aim of our trial is to prove whether or not exercise has any benefit to the life of these patients.

Methods: The study was an open-label, randomized clinical trial. The patients were selected according to the Consolidated Standard of Reporting Trials criteria. The study was registered at the International Standard Randomized Controlled Trial registry (ISRCTN) with the study ID ISRCTN1087174. The study was approved by the Bioethics and Deontology Committee, Medical School of Aristotle University, Thessaloniki (ref: 166/29.10.2015).
\end{abstract}

Results: Once the allocation and the follow-up were completed, 21 patients in the exercise group and 22 in the control group were analyzed. There was no statistical difference between the two groups regarding co-morbidities and disease characteristics; however, the quality of life and the total status of health were superior in the exercise group.

Conclusions: Exercise can improve the quality of life in patients after complex operations like pancreatectomy.

Keywords: Aerobic exercise; Pancreatectomy; Quality of life; Clinical trial; Random allocation

\section{Introduction}

Pancreatic adenocarcinoma is the fourth cause of cancer-re-

Manuscript submitted October 31, 2018, accepted November 16, 2018

aDepartment of Surgery, Agios Dimitrios General Hospital, Thessaloniki, Greece bSchool of Physical Education and Sports Sciences at Serres, "Aristotle" University, Thessaloniki, Greece

cDepartment of Surgery, "Hippokration” University Hospital, Thessaloniki, Greece

dDepartment of Surgery, AHEPA University Hospital, Thessaloniki, Greece

${ }^{e}$ Corresponding Author: Anastasios Katsourakis, Department of Surgery, Agi-

os Dimitrios General Hospital, Thessaloniki, Greece.

Email: tasoskatsourakis@hotmail.com

doi: https://doi.org/10.14740/jocmr3675 lated deaths in the United States. The aggressiveness of this cancer is confirmed by an incidence rate almost equal to its mortality rate, with median survival rates of 3 to 4 months and a 5 -year overall survival rate of $5 \%$. The cornerstone of curative treatment of pancreatic adenocarcinoma is radical pancreatic tumor resection [1-4].

Pancreatic surgery, mainly pancreaticoduodenectomy, is a technically challenging surgical procedure with a high morbidity rate and prolonged length of stay, even in high volume hospitals [5]. Exercise helps patients to not only live longer but also better. In addition to making the circulatory system and muscles stronger, it is known that it can improve mental and emotional functioning and boost productivity. However, until now, there has been no trial that confirms the importance of exercise to patients after pancreas resection. The aim of this single-centre, prospective, randomized trial was to assess the ability of aerobic exercise to improve the quality of life (QOL) in operated patients with pancreatic pathology.

\section{Materials and Methods}

\section{Study design}

The study was an open-label, randomized, prospective, comparative clinical trial. All consecutive patients who underwent pancreatectomy were enrolled in the study, except for those with the following characteristics: 1) Age older than 75 years; 2) American society of Anaesthesiologists (ASA) score greater than 2; 3) Presence of distant metastases; 4) An unresectable tumor; and 5) Pregnancy. The patients were selected according to the Consolidated Standard of Reporting Trials criteria. The study was approved by the Bioethics and Deontology Committee, Medical School of Aristotle University, Thessaloniki (October 29, 2015; ref: 166/29.10.2015). It was monitored by the scientific committee and registered at ISRCTN with the study ID ISRCTN1087174.

The endpoint of the study was the collection of a statistically significant number of patients. Between May 2014 and June 2016, 43 patients anticipating a pancreatectomy were recruited into this study.

The primary outcome measures were blood glucose, $\mathrm{HbA} 1 \mathrm{c}$, albumin and uric acid levels 12 weeks after the initiation of exercise (measured once every 4-week interval).

Additionally, the secondary outcome measure was the 


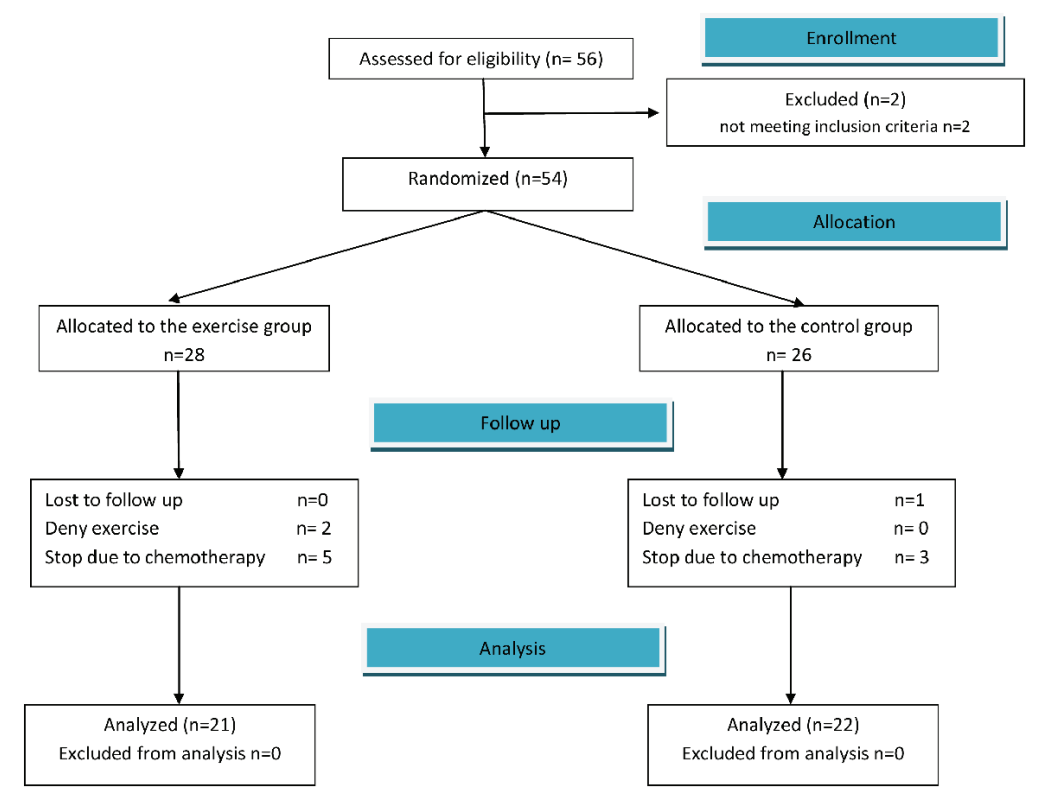

Figure 1. Randomization (flow diagram) according to Consolidated Standard of Reporting Trials.

QOL in both groups 12 weeks after the initiation of exercise according to a questionnaire by the European Organisation for Research and Treatment of Cancer (EORTC QLQ-30) translated into the Greek language [6]. There was no sponsor or funder for this trial.

\section{Patients}

The patients who met the preliminary criteria were randomly assigned into two groups: the treatment group and the control group. Patients in both groups had a pancreatectomy. The surgical procedure was open, and depending on the location of the tumor, the patient underwent a Longmire-Traverso/Whipple operation (pancreatoduodenectomy) or a distal pancreatectomy without the preservation of the spleen. The participants in the intervention group started to exercise 4 weeks after the operation, which involved $30 \mathrm{~min}$ on a static bicycle $(60 \%$ of maximum heart rate) for 12 weeks, three times per week. The participants in the control group did not exercise.

The patients were treated according to the ethical guidelines of the Declaration of Helsinki. All participants gave informed consent to participation before enrolment. The operations were performed and all data were collected at the hospital-based Surgical Department at the Agios Dimitrios General Hospital, Thessaloniki, Greece. All patients were operated on by the same surgical team using the same technique and type of materials.

\section{Statistical analysis}

Data consisted of numerical and categorical variables corresponding to patients' characteristics. The numerical variables were expressed in the form of the mean \pm standard deviation, whereas the categorical variables were expressed as frequency (i.e., in a percentage).

Non-parametric statistical tests were used because the sample size is small. In particular, Fisher's exact test for comparing proportions of categorical data, the Mann-Whitney $\mathrm{U}$ test for comparing continuous, non-normally distributed data and the Friedman test for comparing continuous, non-normally distributed patients' data were conducted repeatedly at $1,2, \ldots$, $\mathrm{t}$ time points, where $\mathrm{t}>2$.

Box plots or histograms were used in order to represent the data sets and to graphically confirm the findings of the inferential statistics. The common significance level of 0.05 was used in all analyses. SPSS 22.0 (IBM Corp. Released, 2013) and R (R Core Team, 2018) were used for all statistical analyses and graphs.

\section{Results}

Between May 2014 and June 2016 (26 months), 56 consecutive patients anticipating pancreatectomy mainly due to pancreatic cancer were recruited into the study. After the exclusion of two patients because they failed to meet the inclusion criteria, 54 were randomized, but finally only 43 were analyzed (Fig. 1). Once the allocation and the follow-up were completed, 21 patients were analyzed for the exercise group and 22 for the control one.

According to the demographics, 21 males and 22 females were included in the study, and the mean age was 59.9 years for the exercise group and 69.1 years for the control group ( $\mathrm{P}$ $=0.003)$, this may lead to lessened comparability of the two groups and can be explained most probably due to the low number of participants. There was no statistical difference be- 
Table 1. Comparison of Demographics, Preoperative and Intraoperative Characteristics Among the Two Groups

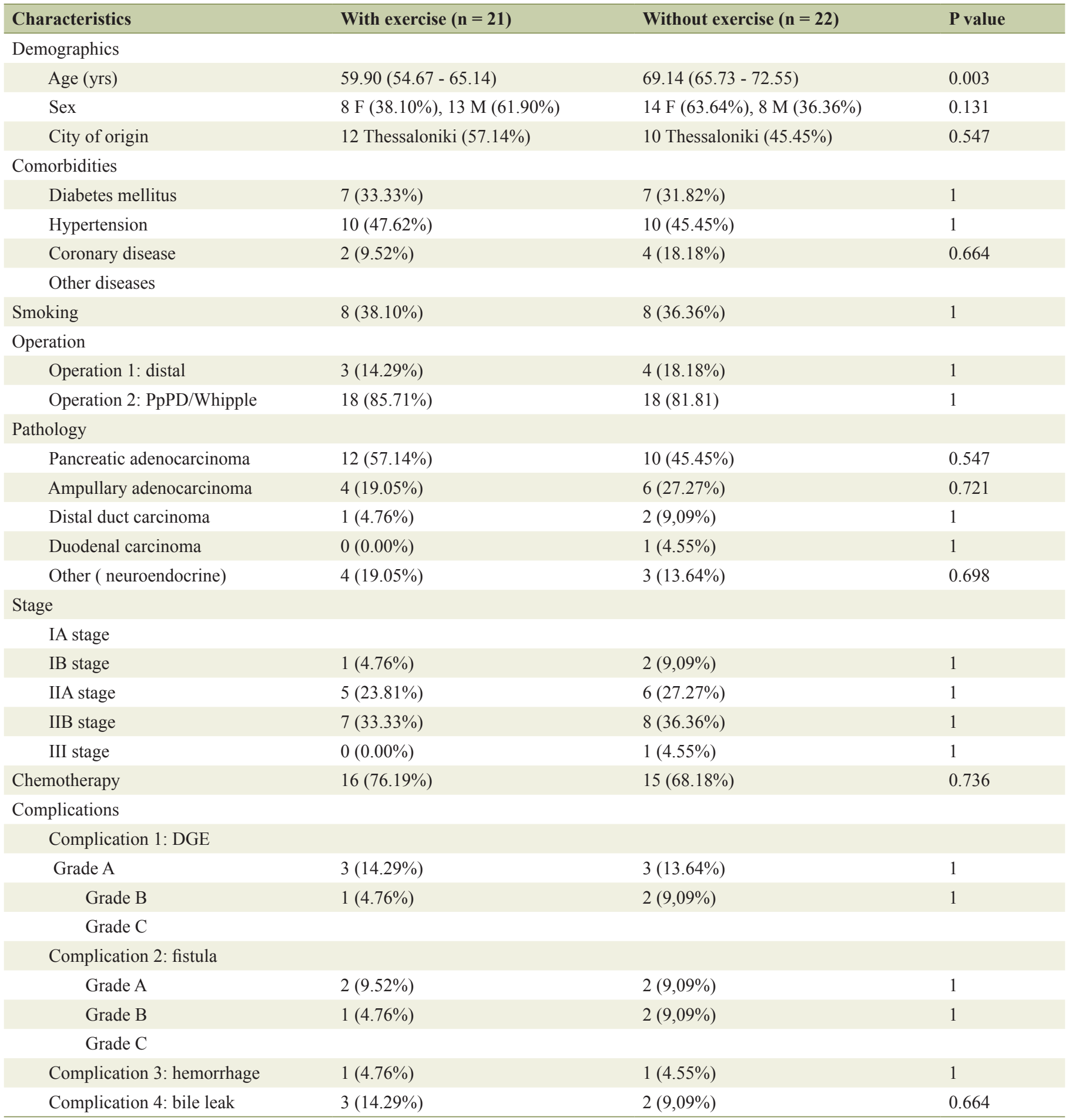

F: female; M: male; DGE: delayed gastric emptying.

tween the two groups regarding co-morbidities and the disease characteristics, which are presented in Table 1.

Concerning the stage of the disease, there was no difference, and almost $70 \%$ of the patients received adjuvant chemotherapy. No patient died after the operation, while postopera- tive complications were observed in 23 patients $(53.5 \%)$ but with no statistical value (Table 1).

After the end of the exercise protocol, the patients from both groups were assessed for their QOL by using the EORTC QLQ-30 questionnaire. 
Table 2. Independent Samples Mann-Whitney Test Grouped by Exercise

\begin{tabular}{llll}
\hline Variables & Sample size & Mann-Whitney U test & P value \\
\hline Health status during the last week & 43 & 99.5 & 0.001 \\
Total status of health & 43 & 311,00 & 0.036 \\
Quality of life & 43 & 417.5 & 0 \\
\hline
\end{tabular}

Using the Mann-Whitney U test (independent samples), there was a strong statistical difference between the exercise group and the control group (Table 2). The QOL, the total status of health as well as the health status during the last week (according to the QLQ-30) were superior in the exercise group (Figs. 2-4).

\section{Discussion}

Pancreatic cancer is among the deadliest malignancies despite being responsible for only $3 \%$ of all new cancer diagnoses. It is expected to be the second most common cause of death by 2030.The reported incidence is highest in developed countries, which likely reflects accuracy of diagnoses in those countries, with Europe bearing a significant part of the burden. The life expectancy at diagnosis is 4.6 months compared to 15.1 years for an age-matched healthy population. Annually, 610,000 915,000 quality-adjusted life years (QALYs) are lost to pancreatic cancer in Europe [7-10].

Pancreatic resection provides the only chance of longterm survival. However, pancreaticoduodenectomy of the pan- creatic head, neck and uncinate process is still a challenging procedure. In high-volume centres, the mortality rate is around $2-4 \%$ and the morbidity rate ranges from $40-58 \%$, which may influence the recovery of patients and their QOL. QOL is a critical outcome in clinical trials and a significant predictor of treatment response. Reviews show that QOL predicts cancer survival above and beyond demographic and medical factors [11-13].

The relationship between exercise and improved physical and psychological health has been well established. The benefits apply to healthy people as well as those with chronic illnesses, such as cancer. The potential benefits associated with exercise in people with cancer include less symptomatology, enhanced functional capacity and improved physical and psychological well-being [14-16].

The benefits of physical activity are evident not only in healthy people but also in patients. Observational and randomized trials have shown that regular physical activity contributes to the treatment of several chronic diseases. There is evidence for prescribing exercise in the primary and secondary prevention of pulmonary and cardiovascular diseases, meta-

\section{Exercise $\mathbf{=}$ no}

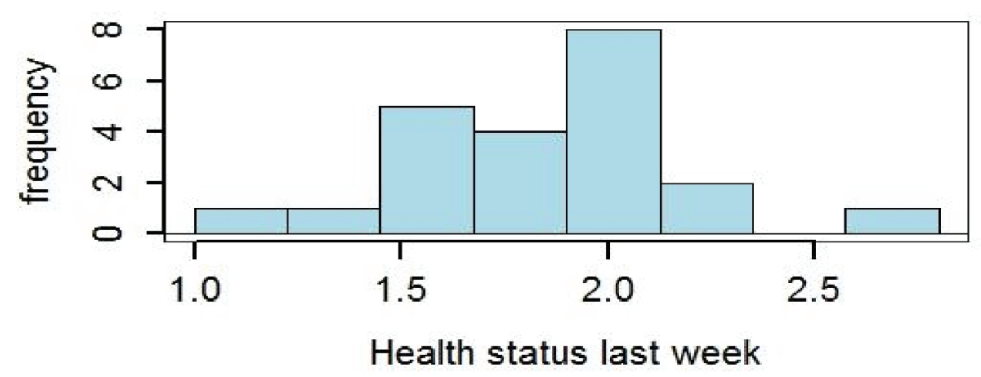

Exercise $=$ yes

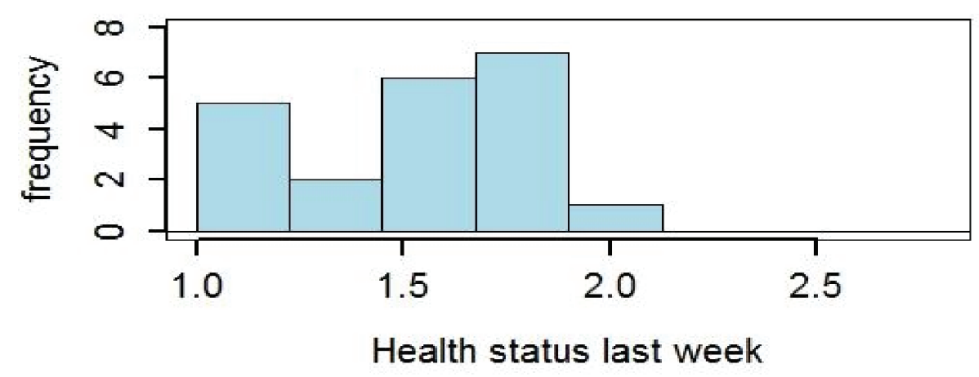

Figure 2. Comparison of health status (during the last week) between the two groups using the EORTC QLQ -30 (the lower number the better). 

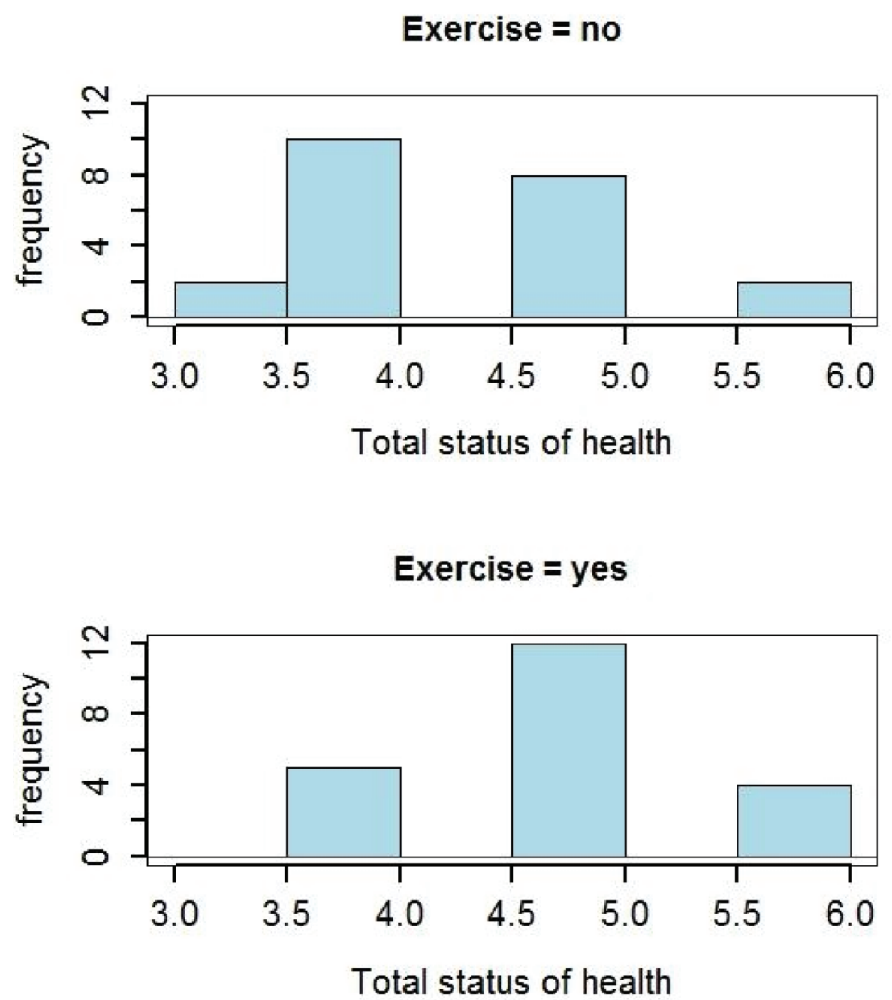

Figure 3. Comparison of total status of health between the two groups using the EORTCQLQ-30 (the highest number the better).
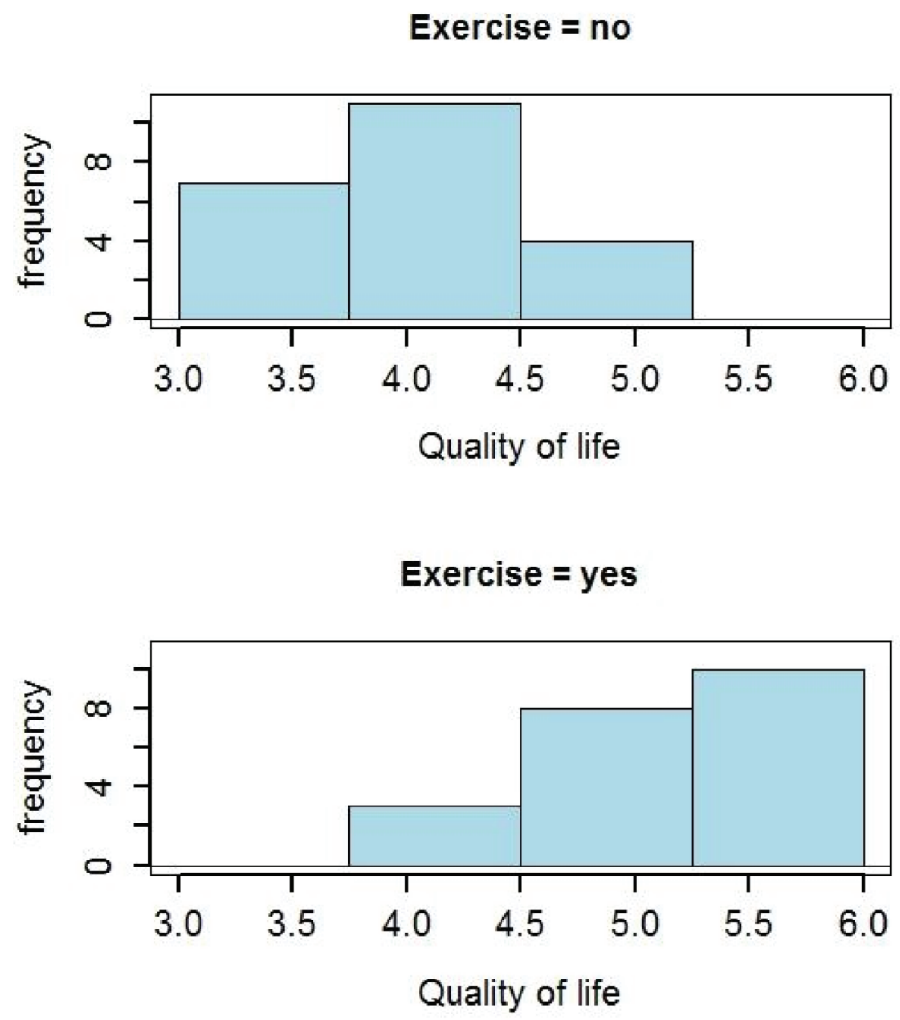

Figure 4. Comparison of the quality of life between the two groups (the highest number the better). 
bolic disorders, muscle, bone and joint diseases, cancer and depression [17-19].

Based on the above facts, we have tried to prove if there is any benefit from exercising for patients after pancreatectomies in our study. We used aerobic exercise and not resistance exercise in order for the activity to be more attractive to the patients and for it to be more accurately monitored at our physiotherapy department. The exercise program included $30 \mathrm{~min}$ on a static bicycle ( $60 \%$ of maximum heart rate) for 12 weeks, starting 1 month after the pancreas resection.

We adopted the EORTC QLQ-30, a cancer-specific questionnaire. It consists of 30 questions that evaluate physical functioning, social functioning, emotional functioning, role functioning, cognitive functioning, nausea and vomiting, pain, fatigue, global health and QOL, insomnia, loss of appetite, constipation, diarrhoea, dyspnoea and financial difficulties.

The analysis of the results was based on nonparametric tests (e.g., the Mann-Whitney U test), mainly due to the low sample size. The analysis demonstrated that exercise can improve QOL with statistical significance.

Despite its interesting outcomes, this study has several limitations. One of them is the low number of participants because it is not easy for everyone to exercise after an operation for practical purposes as well as due to social and personal beliefs. This is why we have used nonparametric tests in order to achieve a lower bias. Additionally, the study was a single-centre study. For this reason, it is necessary to have larger, multicentre trials, which will help to extract safer and more valid results. However, there are some strong points; these are the structure of the trial, which was randomized, as well as its unique focus.

\section{Conclusions}

Exercise plays a very important role in our daily life, which is becoming more stressful and demanding over the years. Our study has proved that exercise improves the QOL in patients after large operations characterised by high morbidity like pancreas resections. This result may change the way we treat and manage these patients in the future.

\section{Acknowledgments}

We would like to express our sincere appreciation to George Karavasilis, Department of Business Administration Technological Educational Institute of Central Macedonia, Greece for his contribution to the statistical analysis.

\section{Conflict of Interest}

The authors declare no conflict of interest.

\section{References}

1. Ouaissi M, Turrini O, Hubert C, Louis G, Gigot JF, Ma- brut JY. Vascular resection during radical resection of pancreatic adenocarcinomas: evolution over the past 15 years. J Hepatobiliary Pancreat Sci. 2014;21(9):623-638.

2. Jemal A, Siegel R, Xu J, Ward E. Cancer statistics, 2010. CA Cancer J Clin. 2010;60(5):277-300.

3. Rau BM, Moritz K, Schuschan S, Alsfasser G, Prall F, Klar E. R1 resection in pancreatic cancer has significant impact on long-term outcome in standardized pathology modified for routine use. Surgery. 2012;152(3 Suppl 1):S103-111.

4. Delpero JR, Bachellier P, Regenet N, Le Treut YP, Paye F, Carrere N, Sauvanet A, et al. Pancreaticoduodenectomy for pancreatic ductal adenocarcinoma: a French multicentre prospective evaluation of resection margins in 150 evaluable specimens. HPB (Oxford). 2014;16(1):20-33.

5. Haga Y, Wada Y, Saitoh T, Takeuchi H, Ikejiri K, Ikenaga M. Value of general surgical risk models for predicting postoperative morbidity and mortality in pancreatic resections for pancreatobiliary carcinomas. J Hepatobiliary Pancreat Sci. 2014;21(8):599-606.

6. Kyriaki M, Eleni T, Efi P, Ourania K, Vassilios S, Lambros V. The EORTC core quality of life questionnaire (QLQ-C30, version 3.0) in terminally ill cancer patients under palliative care: validity and reliability in a Hellenic sample. Int J Cancer. 2001;94(1):135-139.

7. Siegel R, Naishadham D, Jemal A. Cancer statistics, 2013. CA Cancer J Clin. 2013;63(1):11-30.

8. Rahib L, Smith BD, Aizenberg R, Rosenzweig AB, Fleshman JM, Matrisian LM. Projecting cancer incidence and deaths to 2030: the unexpected burden of thyroid, liver, and pancreas cancers in the United States. Cancer Res. 2014;74(11):2913-2921.

9. Parkin DM, Bray F, Ferlay J, Pisani P. Global cancer statistics, 2002. CA Cancer J Clin. 2005;55(2):74-108.

10. Carrato A, Falcone A, Ducreux M, Valle JW, Parnaby A, Djazouli K, Alnwick-Allu K, et al. A systematic review of the burden of pancreatic cancer in europe: real-world impact on survival, quality of life and costs. J Gastrointest Cancer. 2015;46(3):201-211.

11. Heerkens HD, van Berkel L, Tseng DSJ, Monninkhof EM, van Santvoort HC, Hagendoorn J, Borel Rinkes IHM, et al. Long-term health-related quality of life after pancreatic resection for malignancy in patients with and without severe postoperative complications. HPB (Oxford). 2018;20(2):188-195.

12. Heerkens HD, Tseng DS, Lips IM, van Santvoort HC, Vriens MR, Hagendoorn J, Meijer GJ, et al. Health-related quality of life after pancreatic resection for malignancy. Br J Surg. 2016;103(3):257-266.

13. Eaton AA, Gonen M, Karanicolas P, Jarnagin WR, D'Angelica MI, DeMatteo R, Kingham TP, et al. Healthrelated quality of life after pancreatectomy: results from a randomized controlled trial. Ann Surg Oncol. 2016;23(7):2137-2145.

14. Hacker E. Exercise and quality of life: strengthening the connections. Clin J Oncol Nurs. 2009;13(1):31-39.

15. Courneya KS. Exercise in cancer survivors: an overview of research. Med Sci Sports Exerc. 2003;35(11):1846-1852.

16. Courneya KS, Friedenreich CM, Quinney HA, Fields AL, 
Jones LW, Fairey AS. A randomized trial of exercise and quality of life in colorectal cancer survivors. Eur J Cancer Care (Engl). 2003;12(4):347-357.

17. Dunn AL, Trivedi MH, O'Neal HA. Physical activity dose-response effects on outcomes of depression and anxiety. Med Sci Sports Exerc. 2001;33(6 Suppl):S587-597; discussion 609-510.

18. Gomez-Cabrera MC, Domenech E, Vina J. Moderate exercise is an antioxidant: upregulation of antioxidant genes by training. Free Radic Biol Med. 2008;44(2):126-131.

19. Goodyear LJ. The exercise pill - too good to be true? N Engl J Med. 2008;359(17):1842-1844. 